\title{
Bowel function, mental health, and psychosocial function in adolescents with Hirschsprung's disease
}

Trond H Diseth, Kristin Bjørnland, Torunn S Nøvik, Ragnhild Emblem

\begin{abstract}
Congenital intestinal malformations are uncommon and may pose lasting somatic difficulties. Patients with anorectal anomalies have a high frequency of persistent faecal dysfunction and psychosocial problems. This study examined whether adolescents with Hirschsprung's disease have more psychosocial problems than their healthy peers. Nineteen adolescents (mean age 15.7 years) with Hirschsprung's disease were assessed for bowel function, anorectal physiology, mental health, and psychosocial functioning by physical examinations, semistructured interview, and standardised questionnaires. The adolescents were compared with controls. The parents of 13 adolescents with Hirschsprung's disease were interviewed and completed questionnaires. Thirty two per cent of the adolescents with Hirschsprung's disease had significant impairment of continence, but no more psychopathology (16\%) nor psychosocial dysfunction as a group than their healthy peers. Faecal incontinence was associated with poorer psychosocial functioning and parental criticism. The fact that a significant number of patients with Hirschsprung's disease have incontinence into adulthood indicates the need for parental counselling, encouraging realistic expectations about continence.

(Arch Dis Child 1997;76:100-106)
\end{abstract}

Keywords: Hirschsprung's disease; long term follow up; faecal incontinence; psychosocial function.

National Hospital, Oslo: Division of Child and Adolescent

Psychiatry

T H Diseth

Department of Paediatric Surgery K Bjørnland

R Emblem

National Centre for

Child and Adolescent

Psychiatry, Oslo,

Norway

T S Nøvik

Correspondence to:

Dr Trond H Diseth, Division

of Child and Adolescent

Psychiatry, National

Hospital, 0027 Oslo, Norway.

Accepted 2 September 1996 problems in adolescents with both high and low anorectal anomalies. ${ }^{6-11}$ Soiling, staining, and fear of flatus were associated with psychi- atric and psychosocial dysfunction ${ }^{6}$ and incontinence had a negative impact on the family. ${ }^{7}$

Some follow up studies of children with Hirschsprung's disease report a low frequency of incontinence. ${ }^{12-14}$ Others report that different grades of incontinence are relatively common after the Duhamel operation. ${ }^{15}{ }^{16}$ Children with Hirschsprung's disease undergo a number of diagnostic procedures, operations including enterostomy, and hospital admissions. These factors, as well as defecation problems, may pose a threat to their emotional and psychosocial wellbeing. We know, however, of no published studies assessing the psychosocial outcome of adolescents with Hirschsprung's disease and their families.

The study reported here sought to determine whether adolescents with Hirschsprung's disease have more psychosocial problems than their healthy peers. We examined somatic status, mental health, and psychosocial adjustment in adolescents with Hirschsprung's disease and their families, and compared psychosocial and psychiatric problems with a population based peer group. We evaluated the experiences of parents with practical treatment procedures and information, and explored possible associations between somatic and psychosocial variables.

\section{Patients and methods}

PATIENTS

We randomly selected 22 adolescents operated on with the Duhamel technique ${ }^{12}$ from 32 consecutive patients with Hirschsprung's disease born between 1973 and 1983. Three (14\%) of the 22 patients refused to participate. There were no significant differences in age, sex, or bowel function between the participants and non-participants.

We examined and interviewed 19 patients (13 boys, six girls) with a median age of 16 years (range 10-20 years). The parents of 13 of these patients were interviewed and completed questionnaires regarding the behaviour of their child.

The most common proximal level of aganglionosis was the rectosigmoid or sigmoid colon (64\%) and three patients $(16 \%)$ had total colonic aganglionosis. Sixteen $(84 \%)$ had an enterostomy at a median age of 2 months (range 3 days -3 years) and the pull through procedure was performed at a median of 7 months (range 1-60 months). Three patients had the Duhamel operation without an enterostomy. The Duhamel procedure involves the 
Table 1 Demographic characteristics, hospital admissions, and follow up treatment procedures in adolescents with Hirschsprung's disease and a population group

\begin{tabular}{|c|c|c|c|}
\hline \multirow[b]{2}{*}{ Median (range) age (years) } & \multicolumn{2}{|c|}{$\begin{array}{l}\text { Patients with Hirschsprung's } \\
\text { disease }(n=19)\end{array}$} & \multirow{2}{*}{$\begin{array}{l}\begin{array}{l}\text { Population } \\
\text { group }(n=33)\end{array} \\
15(14-17)\end{array}$} \\
\hline & 16 & $(10-20)$ & \\
\hline No (\%) boys & 13 & (68) & $17(52)$ \\
\hline \multicolumn{4}{|l|}{ Family composition } \\
\hline No (\%) with both biological parents & 17 & (89) & $22(67)$ \\
\hline No (\%) with single biological parent & 2 & (11) & $11(33)$ \\
\hline \multicolumn{4}{|l|}{ Median (range) parental education (years) } \\
\hline Mother & 13 & $(9-15)$ & $14(9-17)$ \\
\hline Father & 12 & $(9-19)$ & $14(9-17)$ \\
\hline \multicolumn{4}{|l|}{ No $(\%)$ community } \\
\hline Urban & 9 & $(47)$ & $18(55)$ \\
\hline Rural & 10 & (53) & $15(45)$ \\
\hline Median (range) age at diagnosis (days) & 75 & $(1-1080)$ & - \\
\hline \multicolumn{4}{|l|}{ Hospital admissions } \\
\hline Median (range) No of hospital admissions & 4 & $(2-11)$ & - \\
\hline Median (range) longest hospital stay (days) & 19.5 & $(11-150)$ & - \\
\hline $\begin{array}{l}\text { Median (range) age at last hospital admission } \\
\text { (years) }\end{array}$ & & $(1-16)$ & - \\
\hline \multicolumn{4}{|l|}{ No (\%) having follow up treatment } \\
\hline Extra crushing clamps & 7 & (37) & - \\
\hline Weekly lavage & 3 & (16) & - \\
\hline
\end{tabular}

creation of a retrorectal side to side anastomosis between the posterior wall of the aganglionic rectum and the anterior wall of the pulled through normal colon. The colorectal septum is divided using crushing clamps. The clamps are inserted through the anus and left there for a median of five days. The enterostomy was closed on average 1.5 months after the Duhamel procedure.

The baseline demographic characteristics of the sample, the age at diagnosis, hospital admissions, and the follow up treatment procedures are presented in table 1.

In seven $(37 \%)$ patients an extra procedure with crushing clamps was performed at an average age of 1.5 years (range 1-6 years) because of a residual septum. Another three $(16 \%)$ patients had weekly lavages instituted up to an average age of 3 years (range 0.5-5 years) because of severe constipation. None of the patients had permanent colostomies.

COMPARISON GROUPS

The comparison group for the psychiatric and psychosocial assessments in this study was a subsample drawn from a Norwegian epidemiological study of mental health. The study used a stratified random sampling procedure and a two stage design. ${ }^{17}{ }^{18}$ In the first stage, screening was carried out by means of a standardised questionnaire, the Child Behavior Checklist (CBCL). In the second stage, 49 adolescents, a random sample from the age group 13-15 years of screen negative (CBCL T score $<60$ ) and screen positive (CBCL $\mathrm{T}$ score $\geqslant 60$ ) adolescents in the same proportion as in the screened general population sample, were invited to participate in a more detailed assessment with semistructured interviews of both adolescents and parents. A $\mathrm{T}$ score of 60 , according to the American norm, corresponds to the 95 th centile of the frequency distribution for this age group. Of these 49, 33 adolescents (67\%) (17 boys, 16 girls), mean age 15 years (range 14-17 years), and their parents participated and are referred to as the population group. There was no significant difference in CBCL mean total behaviour problem score between participants and non-participants in the second phase. The population group and the group of patients with Hirschsprung's disease who completed the same questionnaires and interviews were similar with respect to age $(\mathrm{U}=278.5, \mathrm{p}=0.49)$, sex $\left(\chi^{2}\right.$ test with Yates's correction $\left.\left(\chi_{y}^{2}\right)=0.84, \mathrm{df}=1, \mathrm{p}=0.36\right)$ and sociodemographic characteristics (table 1 ).

The control group for the assessments of bowel function and anal canal manometry was a sample of 14 patients (seven boys and seven girls). The mean age was 16 years (range 10-20 years). These patients were admitted to hospital with urological diseases or abdominal pain and had no reported anorectal disease or dysfunction. The patient group and the controls for the somatic assessments were not different with respect to age $(\mathrm{U}=126.0, \mathrm{p}=$ $0.82)$ or $\operatorname{sex}\left(\chi_{\mathrm{y}}^{2}=0.50, \mathrm{df}=1, \mathrm{p}=0.48\right)$.

SOMATIC ASSESSMENT

Anorectal function was assessed in patients and somatic controls by clinical and physiological examination..$^{19}$ Faecal continence function was graded according to the Wingspread classification, ${ }^{20}$ rating four clinical states of continence: (1) clean, (2) staining, (3) intermittent faecal soiling, and (4) constant soiling. Constipation was defined as having fewer than three bowel movements each week or needing regular laxatives, or both. Flatus continence function was assessed by asking the patients and the controls to indicate their control of flatus on a $10 \mathrm{~cm}$ visual analogue scale with no numbers or categories provided. End points were defined as 'very certain' (0) and 'very uncertain' (10). Anal canal manometry was performed with a $2 \mathrm{~mm}$ microtransducer measuring the anal canal resting pressure and the increase in pressure during maximum squeeze.

PSYCHIATRIC ASSESSMENT

The psychiatric assessment of the adolescents was based on the semistructured interview Child Assessment Schedule (CAS). ${ }^{21}{ }^{22}$ The reliability and validity of the CAS is well established. ${ }^{23}$ The questions generate scores for 11 topic areas of the patient's life: school, friends, activities, family, fears, worries and anxieties, self image, mood, physical complaints, expression of anger, and reality testing symptomatology. Answers scoring 1 (symptoms or problems present) provide a total problem score. Another set of scores consists of symptom complexes analogous to psychiatric diagnoses in the Diagnostic and Statistical Manual of Mental Disorders (DSM-III-R). ${ }^{24}$

The interviews were supplemented with semistructured questions covering the adolescents' views of the present situation regarding their intestinal malformation.

The adolescents also completed the standardised questionnaire Youth Self-Report (YSR), ${ }^{25}$ and one of the parents completed the CBCL. ${ }^{26}$ The reliability and validity of the questionnaires has been demonstrated in both clinical and non-clinical populations and across cultures. ${ }^{27}$ For both forms the questions are scored 0 (no), 1 (sometimes), and 2 (often). Eight syndrome scales are generated and 
subdivided into two subscales. The internalising subscale includes withdrawal, somatic complaints, and anxiety/depression. The externalising subscale includes delinquent and aggressive behaviour. Norms for these standardised questionnaires in the Scandinavian countries are not yet established. According to the American norm, the 90th centile is used as a cut off point for total, internalising, and externalising scores implying psychiatric problems.

ASSESSMENT OF ADOLESCENT PSYCHOSOCIAL FUNCTION

The Children's Global Assessment Scale $(\mathrm{CGAS})^{28}$ was used to assess the overall psychosocial function of the adolescents. The reliability, discriminant validity, and clinical usefulness of the CGAS are well documented. ${ }^{29}{ }^{30}$ Psychosocial problems of clinical significance are reflected by a score less than 71 . The CGAS was scored on information from the child interview and was blind to the child's somatic function. The score ranges from 100 (excellent function) to 0 (severe malfunction).

PARENTAL ASSESSMENT

Parents were interviewed separately $(n=13)$ by a child psychiatrist using a slightly modified version of the Parental Account of Children's Symptoms (PACS) ${ }^{31}$ to obtain information on the children's behaviour and emotional problems as seen by their parents. The PACS is a standardised, semistructured, investigator based interview which includes an interviewer assessment of the warmth and criticism of the parents towards the index child during the interview, adapted from the parent interviews in the expressed emotion research tradition in relation to adult psychopathology. ${ }^{32}$ Warmth is rated on a four point scale from 0 (a great deal of expressed warmth) to 3 (little or no demonstration of warmth throughout the interview). Criticism is scored on a five point scale from 0 (no expressed criticism) to 4 (a lot of expressed criticism throughout the interview).

The parent interviews were supplemented with semistructured questions covering parental experiences and concerns regarding the child and siblings, the emotional and practical aspects of treatment procedures, and medical follow up.

ASSESSMENT OF CHRONIC FAMILY DIFFICULTIES A global assessment of chronic family difficulties (CFD) $)^{34}{ }^{35}$ was based on information from the parental interview about the present family situation - for example, finances, housing, employment, network support, and possible marital and family discord, as well as the physical and mental health of all the family members. The term 'chronic' implies that these difficulties had lasted for more than one year and were still present at the time of the interview. Chronic family difficulties was scored on an interval scale from 0 to 6 , where 0 implies none and 6 implies very severe family strain or difficulties.
ASSESSMENT OF INTER-RATER RELIABILITY

The psychiatric and psychosocial assessments were administered by the first author to the Hirschsprung's disease group and by the first and the third author to the population group. The adolescent and parent interviews in both the Hirschsprung's disease and population groups were videoed for testing of inter-rater reliability. Assessment of inter-rater reliability of CAS and CGAS was based on 20 randomly selected videoed interviews of the population group and 10 videoed patient interviews. To assess the inter-rater reliability of PACS and CFD, 19 videoed parental interviews of the population group and eight videos of PACS interviews in the patient group were randomly selected. The videoed interviews were independently scored by a child psychiatrist blind to other measures in the assessment and the diagnosis.

In the population group the intraclass correlation (ICC 1.1) for the CAS total symptom score was 0.94 and 0.86 for the CGAS. The $\kappa$ correlation coefficients (weighted) were 0.53 for warmth and 0.48 for criticism.

In the patient group the intraclass correlation (ICC 1.1) for the CAS total symptom score was 0.94 and 0.91 for the CGAS. The $\kappa$ correlation coefficients (weighted) were 0.71 for warmth and 0.65 for criticism. Intraclass correlation (ICC 1.1) was 0.92 for the CFD scores.

STATISTICS AND ETHICS

The results are reported as medians and ranges unless otherwise stated. Non-parametric statistics were used for non-normally distributed variables. For the assessment of differences between independent groups, a $\chi^{2}$ test with Yates's correction $\left(\chi_{\mathrm{y}}^{2}\right)$ or Fisher's exact test for small samples was used for categorical variables and the Mann-Whitney $U$ test for continuous variables. Dependency between sets of variables is expressed through the nonparametric Spearman's rank correlation coefficient $\left(r_{s}\right)$. A linear multiple regression analysis was performed to determine independent somatic and family/parental correlates of the CGAS contributing significantly to the variance of CGAS. ${ }^{36} \mathrm{~A}$ two tailed $\mathrm{p}$ value less than 0.05 was considered statistically significant unless otherwise stated. All calculations were performed using the SPSS statistics program for Windows.

Informed written consent was obtained from both the adolescents and the parents. The study was approved by the Regional Ethics Committee for Medical Research.

\section{Results}

SOMATIC STATUS

Continence function and bowel function in the patient group and controls are given in table 2 . The six patients $(32 \%)$ who had faecal dysfunction at the time of assessment were all staining (grade 2 ). Three patients (16\%) and two controls $(14 \%)$ felt unsure about passing wind (visual analogue scale $>3.0$ ). Continence function was not associated with age or sex. There was a significant difference in faecal 
Table 2 Continence function, constipation, and anorectal pressures of adolescents with Hirschsprung's disease and controls

\begin{tabular}{|c|c|c|}
\hline & $\begin{array}{l}\text { Patients with Hirschsprung's } \\
\text { disease }(n=19)\end{array}$ & Controls $(n=14)$ \\
\hline \multicolumn{3}{|l|}{ Faecal continence function $(\text { No }(\%))^{\star}$} \\
\hline Grade 1 & $13(68)$ & $14 \quad(100)$ \\
\hline Grade $2-4$ & $6(32)$ & $0 \ddagger$ \\
\hline \multicolumn{3}{|l|}{ Flatus continence function $\dagger$} \\
\hline VAS 0-3 (No (\%)) & $16(84)$ & $12 \quad(86)$ \\
\hline VAS $4-10(\mathrm{No}(\%))$ & $3(16)$ & 2 (14) \\
\hline Median (range) & $0(0-5)$ & $0.5(0-5)$ \\
\hline No (\%) with constipation & $3(16)$ & 0 \\
\hline \multicolumn{3}{|c|}{ Median (range) anal canal pressures $\left(\mathrm{cmH}_{2} 0\right)$} \\
\hline Resting pressure & $70(40-120)$ & $98 \quad(80-105) \ddagger$ \\
\hline Squeeze pressure & $100(50-200)$ & $200 \quad(90-300) \ddagger$ \\
\hline \multicolumn{3}{|c|}{$\begin{array}{l}\star^{\star} \text { Faecal continence function was graded according to the Wingspread classification: }(1) \text { clean, }(2) \\
\text { staining, (3) intermittent faecal soiling, and (4) constant faecal soiling. } \\
\text { †Flatus continence function was indicated on a } 10 \mathrm{~cm} \text { visual analogue scale (VAS). } \\
\ddagger \text { Significant difference between patients with Hirschsprung’s disease and controls }(p<0.05) \text {. }\end{array}$} \\
\hline \multicolumn{3}{|c|}{$\begin{array}{l}\text { Table } 3 \text { Mental health and psychosocial functioning in adolescents with Hirschsprung's } \\
\text { disease and the population group. Values are median (range) unless otherwise stated }\end{array}$} \\
\hline & $\begin{array}{l}\text { Patients with Hirschsprung's } \\
\text { disease }(n=19)\end{array}$ & $\begin{array}{l}\text { Population group } \\
(n=33)\end{array}$ \\
\hline \multicolumn{3}{|l|}{ Mental health } \\
\hline DSM-III-R diagnosis $(\text { No }(\%))^{\star}$ & $3(16)$ & $4(12)$ \\
\hline Dysthymic disorder & 0 & 1 \\
\hline Major depressive syndrome & 1 & 0 \\
\hline Overanxious disorder & 3 & 1 \\
\hline Conduct disorder & 0 & 2 \\
\hline \multicolumn{3}{|l|}{ CAS scores $\dagger$} \\
\hline Total problem score & $11(6-62)$ & $15(3-64)$ \\
\hline \multicolumn{3}{|l|}{ CBCL raw scores $\ddagger$} \\
\hline Total behaviour score & $9(1-32)$ & $7(0-76)$ \\
\hline Internalising score & $3(0-11)$ & $4(0-19)$ \\
\hline Externalising score & $2(0-11)$ & $2(0-39)$ \\
\hline \multicolumn{3}{|l|}{ YSR raw scores } \\
\hline Total behaviour score & $24(7-93)$ & $22(0-86)$ \\
\hline Internalising score & $6(0-42)$ & $5(0-30)$ \\
\hline Externalising score & $8(2-15)$ & $7(0-45)$ \\
\hline \multicolumn{3}{|l|}{ Psychosocial fiunctioning } \\
\hline CGAS score $\|$ & $86(54-96)$ & $85(55-98)$ \\
\hline
\end{tabular}

${ }^{\star}$ Diagnostic and Statistical Manual of Mental Disorders. 3rd revised edition.

tChild Assessment Schedule.

¥Child Behavior Checklist.

ๆYouth Self-Report.

\| Children's Global Assessment Scale.

continence function between patients and controls $(p=0.027)$, but no significant difference in flatus control or constipation.

Of the three patients (16\%) who reported constipation, none was staining. The anal canal resting pressure and increments in pressure during squeeze were significantly lower in the patients than in the controls $(\mathrm{p}<0.05)$.

\section{MENTAL HEALTH AND PSYCHOSOCIAL}

FUNCTIONING

We found no significant differences between patient and population groups in the rate of psychiatric diagnoses or scores on the CBCL and YSR. Three $(16 \%)$ of 19 adolescents with Hirschsprung's disease met the DSM-III-R criteria for a psychiatric diagnosis (table 3), one of the adolescents receiving two diagnoses. The psychopathology covered internalising problems: low self esteem, poor body concept, withdrawal, overanxiousness, sadness, or depression. Two of the three adolescents with a psychiatric diagnosis were staining. Both had overanxious disorders. In the population group, none of the four adolescents with a psychiatric diagnosis had any chronic physical problems or illnesses.

The type and number of DSM-III-R diagnoses, the median CAS total symptom score, the median CBCL and YSR total behaviour, externalising and internalising raw scores, and the median CGAS score in patients and population group are given in table 3 .

The CAS total symptom and content area scores were similar for the adolescents with Hirschsprung's disease and the population group, and no significant differences in age or sex were found regarding the CAS scores. The values of CBCL and YSR in the Hirschsprung's disease group were comparable with the population group (table 3).

The adolescents with Hirschsprung's disease had a median score of 86 on the CGAS (table 3 ), which indicates 'well functioning'. Five $(26 \%)$ of the adolescents with Hirschsprung's disease had an impairment of psychosocial function (CGAS <80). All three adolescents who met the DSM-III-R criteria for a psychiatric diagnosis had CGAS scores <71. With regard to mental health and psychosocial function, no significant differences in age or sex were found. There was no significant difference in CGAS scores between the Hirschsprung's disease group and the population group, and we found no significant differences in the CGAS scores between the adolescents of divorced and non-divorced parents in the Hirschsprung's disease group nor in the population group. Between the continent and incontinent patients with Hirschsprung's disease, however, there was a significant difference in CGAS scores $(\mathrm{z}=-2.47, \mathrm{p}=0.014)$. There were no significant differences in CAS or psychosocial function scores between those patients $(n=10)$ where invasive proceduresextra crushing clamps $(n=7)$ or lavages $(n=$ 3) - had been instituted and patients without such follow up treatments. There was, however, a significant difference in CBCL externalising score $(\mathrm{z}=-2.46, \mathrm{p}=0.014)$ and a trend towards a difference with regard to YSR externalising score $(z=-1.65, p=0.09)$ with higher externalising scores in the group with extra invasive procedures.

Eleven $(58 \%)$ of the adolescents with Hirschsprung's disease reported no somatic or psychosocial problems at the time of the assessment. Five (26\%) mentioned worries about incontinence or flatus, four $(21 \%)$ mentioned scars, and two mentioned problems with rumbling.

Fourteen $(74 \%)$ of the adolescents with Hirschsprung's disease could not remember any difficulties related to their anomaly during their lifetime, whereas five (26\%) identified problems before starting school or up to 12 years of age because of emotional difficulties with hiding symptoms of leakage or the long lasting hospital admissions.

The adolescents were asked what advice they would give other parents of children with Hirschsprung's disease. All emphasised openness, the provision of information, and encouraging a normal upbringing with a supportive attitude. In addition, four mentioned respect of his/her boundaries, especially during adolescence. 
PARENTAL AND FAMILY DIFFICULTIES

Differences in the baseline characteristics between the Hirschsprung's disease group and the population group were not statistically significant (table 1). The economic, housing, and educational situations and the physical and mental health of the family members were generally good in the Hirschsprung's disease group.

The Hirschsprung's disease families had, as a group, no or minor chronic family difficulties (mean (SD) $1.9(1.4)$ ). Five $(26 \%)$ families had moderate to severe chronic strain (score 3-6). The type of CFD was mainly due to somatic and mental health problems in the parents or the siblings and to minor intrafamilial conflicts.

No significant differences were found between the Hirschsprung's disease families and the population group with regard to parental warmth (mean (SD) $0.4(0.7) v 0.3(0.5)$ ) and parental criticism (mean (SD) $0.3(0.6) v 0.4$ $(0.5))$.

Three $(23 \%)$ of the parents reported that the weeks of uncertainty before the diagnosis was made were the most difficult part of having a child with Hirschsprung's disease. Another three mentioned the feelings evoked by the invasive procedures of crushing clamps and lavage. Two (15\%) mothers considered as most difficult their early separation from their child when they were admitted to the National Hospital for treatment. They also cited the number and duration of hospital admissions during the first years of life.

When informed about the medical diagnosis, $11(85 \%)$ parents of the children with Hirschsprung's disease recalled that they reacted with relief or recalled no special reaction. Nine $(69 \%)$ of the Hirschsprung's disease parents assessed the initial information on diagnosis and further treatment as satisfactory.

Three $(23 \%)$ of the parents of adolescents with Hirschsprung's disease reported that the problems of the child had influenced their marital relationship and family life, and this was mostly positive-for example, they had become emotionally more close as partners, united by the experience of having a disabled child. There was a difference between the Hirschsprung's disease group and the population group in terms of a threefold greater incidence of divorce in the latter ( $11 v 33 \%)$, but the difference was not statistically significant.

Eleven $(85 \%)$ of the Hirschsprung's disease parents experienced support from family and friends after having a child with Hirschsprung's disease. The anomaly and problems with faecal dysfunction had never been difficult to talk about.

At the time of the interview, three $(23 \%)$ of the Hirschsprung's disease parents still expressed worries about the present somatic and psychosocial situation of their adolescents.

ASSOCIATIONS BETWEEN SOMATIC STATUS, MENTAL HEALTH, AND PSYCHOSOCIAL

FUNCTIONING

Faecal incontinence was significantly correlated with poorer psychosocial function
(CGAS) $\left(r_{s}=-0.58, \mathrm{p}=0.01\right)$ and parental criticism $\left(r_{s}=0.63, \mathrm{p}=0.03\right)$. No significant correlations were found with regard to flatus continence function.

The psychosocial functioning (CGAS) of the adolescents with Hirschsprung's disease was significantly correlated with faecal continence function $\left(r_{s}=-0.58, \mathrm{p}=0.01\right)$, mental health; YSR total score $(r=-0.89, \mathrm{p}<0.0001)$, and family/parental variables such as parental educational level $\left(r_{s}=0.76, \mathrm{p}=0.002\right), \mathrm{CFD}$ $\left(r_{s}=-0.79, \mathrm{p}<0.0001\right)$, and parental warmth $\left(r_{s}=-0.79, \mathrm{p}=0.002\right)$.

To investigate further the relative importance of the independent somatic and family variables in predicting the psychosocial outcome (CGAS), we conducted multiple regression analysis. A stepwise regression analysis with CGAS as the dependent variable and entering both the somatic variable faecal continence function and the family/parental variables parental educational level, CFD, and parental warmth as explanatory variables, revealed that the strongest predictor of CGAS was parental warmth, explaining $71 \%$ of the variance of CGAS $(\beta=-16.9, \mathrm{SE}=3.19, \mathrm{p}=$ $0.0004)$. The somatic and the other family/ parental variables did not contribute significantly to the explained variance of psychosocial functioning of the adolescents.

\section{Discussion}

In spite of the significant impairment of faecal continence, we found that the adolescents with Hirschsprung's disease did not have more mental or psychosocial problems than their peers.

This long term follow up of adolescents with Hirschsprung's disease revealed that $32 \%$ of the adolescents with Hirschsprung's disease operated by the Duhamel technique had moderate faecal continence problems. This accords with other studies ${ }^{15} 16$ indicating that some degree of incontinence is common after the procedure.

Psychosocial functioning was significantly correlated with the degree of current faecal continence. This association between psychosocial function and severity of physical impairment corresponds with findings in other studies of congenital malformations ${ }^{5738}$ and with our findings in patients with anorectal anomalies. $^{7}$ Therefore, when treating and following up patients with Hirschsprung's disease doctors need to focus on the continence function and its impact on psychosocial function. Problems related to leakage seem to be shameful, embarrassing, private, and socially unacceptable, independent of the aetiology of the intestinal dysfunction.

Parental criticism was also significantly correlated with faecal continence. This is in contrast with findings in adolescents with anorectal anomalies, ${ }^{7}$ where there was no relationship. We speculate that adolescents with Hirschsprung's disease, soiling after the expected age for being clean, evoke more criticism because their parents have been told that their child has a good prognosis. The fact that patients with Hirschsprung's disease may 
experience incontinence problems into adolescence should be taken into consideration when counselling parents regarding realistic expectations of the child's toilet training. The motivation for faecal control in patients with Hirschsprung's disease, however, may increase with age and a supportive interaction within the family is important for long term psychosocial functioning.

We found no over-representation of mental and psychosocial problems in the Hirschsprung's disease group when compared with a population based group. Behavioural and emotional scores assessed in questionnaires by the parents and adolescents were similar to the scores of the population sample. As subtle or mild psychological disturbances may, however, be difficult to elicit in questionnaires, ${ }^{49-41}$ specific areas of psychosocial competence were evaluated in the semistructured interview (CAS). These data did not suggest that the adolescents with Hirschsprung's disease were more impaired than the population group. A prevalence rate of psychiatric disorders of $16 \%$ in patients with Hirschsprung's disease is as expected in the population. ${ }^{42}{ }^{43}$ The scores for psychosocial functioning in patients with Hirschsprung's disease indicate psychosocial functioning within the normal range.

A high incidence of divorce may represent a risk factor in terms of the psychosocial adjustment of children. The difference between the Hirschsprung's disease group and the population group in terms of a threefold greater incidence of divorce in the latter could have acted as a confounder for psychosocial function in this study. As we found no significant differences in CGAS between the adolescents of divorced and non-divorced parents in both the Hirschsprung's disease group and the population group, however, the effect of divorce as a confounder seems unlikely.

In the initial screening ${ }^{18}$ and in the interviews the response rates of the population sample were fairly low. The results from the general population study, however, are comparable with and supported by the results from a twin study with a higher response rate. ${ }^{17}$ Furthermore, any biases present in the population group did not present an advantage to the Hirschsprung's disease group.

The good mental health and psychosocial functioning in patients with Hirschsprung's disease contrasted with the high frequency of psychiatric disorders (58\%) and psychosocial problems in adolescents with anorectal anomalies. ${ }^{6}$ Our findings were also surprising considering that other studies of children with long term physical illness ${ }^{13}$ have showed twice the expected rate of psychiatric disorders. Thus we should take into consideration the nature, stage, and severity of the physical illness. ${ }^{4}$ We consider that Hirschsprung's disease can be classified as a long term illness as a substantial number of our patients still had moderate long term continence problems into adolescence. The risk for maladjustment seems to differ between various diagnostic groups, probably related to both somatic, treatment, and psychosocial factors. ${ }^{14}$
The patients with Hirschsprung's disease who had mental and psychosocial problems had emotional disorders with internalising problems: low self esteem, poor body concepts, withdrawal, overanxiousness, or sadness. As expected from other epidemiological studies, ${ }^{42}{ }^{43}$ both emotional and behavioural disorders were found in the population group. This is in accordance with findings from other studies ${ }^{4}$ where children with long term physical illnesses that do not affect cerebral functioning are particularly prone to emotional symptoms rather than antisocial behaviour. We suggest that the symptoms of the current disability have an impact on all the aspects of an adolescent's life experiences, and in the Hirschsprung's disease group the embarrassing and antisocial problems with faecal incontinence may result in emotional problems.

We may question why externalising scores on the youth (YSR) and parental (CBCL) questionnaires seemed to be higher for patients where invasive procedures had been instituted than in patients without such follow up treatments. We speculate that these therapeutic procedures, resulting in pain, a power struggle between the protesting child and the parents performing these procedures, and a negative parent-child relationship led to externalising problems.

Our findings illustrate that a congenital disorder does not necessarily imply that there will be mental and psychosocial problems. Psychological growth and wellbeing can be achieved if the disorder has been properly managed by the child, parents, and health professionals. We found that high parental warmth was the strongest independent predictor of the psychosocial functioning of adolescents with Hirschsprung's disease. Protective and involved parenting may be psychologically adaptive for children under stress and long term illness may lead to heightened maternal empathy towards the child. ${ }^{4}$ The present study indicates that this may counteract other risk factors such as early mother-child separations, hospital admissions, and invasive treatment procedures.

The authors thank Philip Graham and Inger Helene Vandvik for helpful advice, Astrid Aasland for scoring the videos used in helpful advice, Astrid Aasland for scoring the videos used in secretarial assistance, and Medinnova for financial support.

1 Pless IB, Nolan T. Revision, replication and neglectresearch on maladjustment in chronic illness. $\mathcal{f}$ Child Psychol Psychiatry 1991;32:347-65.

2 Rutter M, Graham P, Yule W. A neuropsychiatric study in childhood. Clinics in developmental medicine 35/36. London: Heinemann Medical Books, 1970.

3 Eiser C. Chronic childhood disease: an introduction to psychological theory and research. Cambridge: Cambridge University Press, 1990

4 Garralda ME. Chronic physical illness and emotional disorder in childhood: where the brain's not involved, there may still be problems. Br F Psychiatry 1994;164:8-10.

5 Heller A, Rafman S, Zvagulis I, Pless IB. Birth defects and psychosocial adjustment. Am f Dis Child 1985;139:257-63.

6 Diseth TH, Emblem R. Somatic function, mental health and psychosocial adjustment of adolescents with anorectal anomalies. F Pediatr Surg 1996;31:638-43.

7 Diseth TH, Emblem R, Vandvik IH. Adolescents with anorectal malformations and their families. Family Systems Medicine 1995;13:215-31.

8 Rintala R, Mildh L, Lindahl H. Fecal continence and quality of life in adult patients with an operated low anorectal ity of life in adult patients with an operated
malformation. F Pediatr Surg 1992;27 902-5.

9 Yeung CK, Kiely EM. Low anorectal anomalies: a critical appraisal. Pediatr Surg Int 1991;6:333-5. 
10 Ditesheim JA, Templeton JM Jr. Short-term v long-term quality of life in children following repair of high imperfoquality of life in children following repair
rate anus. 7 Pediatr Surg 1987;22:581-7.

11 Ludman L, Spitz L, Kiely EM. Social and emotional impact of faecal incontinence after surgery for anorectal abnormalities. Arch Dis Child 1994;71:194-200.

12 Duhamel B. A new operation for the treatment of Hirschsprung's disease. Arch Dis Child 1960;35:38.

13 Livaditis A. Hirschsprung's disease: long-term results of the original Duhamel operation. F Pediatr Surg 1981;16:484-6.

14 Rescorla FJ, Morrison AM, Engles D, West KW, Grosfeld JL. Hirschsprung disease. Evaluation of mortality and
long-term function in 260 cases. Arch Surg 1992;127:93442.

15 Marty TL, Seo T, Matlak ME, Sullivan JJ, Black RE, Johnson DG. Gastrointestinal function after surgical correction of Hirschsprung's disease: long-term follow-up in 135 patients. F Pediatr Surg 1995;30:655-8.

16 Heij HA, de Vries X, Bremer I, Ekkelkamps S, Vos A. Longterm anorectal function after Duhamel operation for term anorectal function after Duhamel operation

17 Gjone H, Nøvik TS. Parental ratings of behaviour problems: a twin and general population comparison. $\mathcal{F}$ Child Psychol

18 Nøvik TS. Are postal surveys in child psychiatry feasible? Results of an epidemiological study. Nord $\mathcal{F}$ Psychiatry 1995;49:337-42.

19 Emblem R, Diseth TH, Mørkrid L, Stien R, Bjordal R. Ana endosonography and physiology in adolescents with corrected low anorectal anomalies. F Pediatr Surg 1994;29: 447-51.

20 Stephens FD, Smith ED. Classification, identification, and assessment of surgical treatment of anorectal anomalies. Pediatr Surg Int 1986:1:200-5.

21 Hodges K, Kline J, Stern L, et al. The development of a child assessment interview for research and clinical use. $\mathcal{F}$ Abnorm Child Psychol 1982;10:173-89.

22 Hodges K. Manual for the child assessment schedule (CAS). [Unpublished manuscript.] Columbia: University of Missunpublished 1985.

23 Hodges K, McKnew D, Cytryn L, Stern L, Kline J. The child assessment schedule (CAS) diagnostic interview: a report on reliability and validity. $\mathcal{F}$ Am Acad Child Psychiatry 1982;21:468-73

24 American Psychiatric Association. Diagnostic and statistical manual of mental disorders. 3rd revised edition. Washington DC: American Psychiatric Association, 1987.

25 Achenbach TM, Edelbrock CS. Manual for the youth self report and profile. Burlington: University of Vermont, Department of Psychiatry, 1987.

26 Achenbach TM. Manual for the child behavior checklist and 1991 profile. Burlington: University of Vermont, Department of Psychiatry, 1991.
27 Verhulst FC, Koot HM. Child psychiatric epidemiology. Concepts, methods, and findings. Developmental clinical psychology and psychiatry. Vol 23. Newbury Park: Sage, 1992.

28 Schaffer D, Gould US, Brasic J, et al. Children's global assessment scale (CGAS). Psychopharm Bull 1985;21:7478.

29 Steinhausen HC. Global assessment of child psychopathology. $f$ Am Acad Child Adolesc Psychiatry 1987;2:203-

30 Bird HR, Canino G, Rubrio-Stripee M, Ribera J. Further measures of the psychometric properties of the children's global

31 Taylor E, ed. The overactive child. Clinics in developmental medicine No 97. Oxford: Blackwell Scientific, 1986.

32 Brown G, Birley J, Wing J. Influence of family life on the course of schizophrenic disorders: a replication. $\mathrm{Br} f$ course of schizophrenic disor
Psychiatry 1972;121:241-58.

33 Vaughn C, Leff J. The influence of family and social factors on the course of psychiatric illness; a comparison of schizophrenic patients. Br 7 Psychiatry 1976;129:125-37.

34 Vandvik IH, Høyeraal HM, Fagertun H. Chronic family difficulties and stressful life events in recent onset juvenile arthritis. F Rheumatol 1989;16:1088-92.

35 Bjørnstad P, Lindberg H, Spurkland I. Unge hjerter $i$ faresonen. Oslo: Tano, 1990.

36 Kleinbaum D, Kupper L, Muller K. Applied regression analysis and other multivariable methods. 2nd Ed. Boston: PWS-KENT Publishing, 1988.

37 Sandberg S. Psychiatric disorder in children with birth anomalies. Acta Psychiatrica Scandinavica 1976;54:1-16.

38 Spurkland I, Bjørnstad PG, Lindberg H, Seem E. Mental health and psychosocial functioning in adolescents with congenital heart disease. Acta Paediatr 1993;82:71-6.

39 Verhulst FC, Althaus M, Berden CFMG. The child assessment schedule: parent-child agreement and validity measment schedule: parent-child agreement and validity
ures. f Child Psychol Psychiatry 1987;28:455-66.

40 Perrin EC, Stein REK, Drotar D. Cautions in using the Perrin EC, Stein REK, Drotar D. Cautions in using the about children with a chronic illness. $f$ Pediatr Psychol 1991;16:411-21.

41 Thompson RJ, Merritt KA, Keith BR, et al. Mother-child agreement on the child assessment schedule with nonreferred children: a research note. F Child Psychol Psychiatry 1993;34:813-20.

42 Rutter M. Isle of Wight revisited: twenty-five years of child psychiatric epidemiology. 7 Am Acad Child Adolesc Psychiatry 1989;28:633-53.

43 Brandenburg NA, Friedman RM, Silver SE. The epidemiology of childhood psychiatric disorders: prevalence findings from recent studies. F Am Acad Child Adolesc Psychiatry 1990;29:76-83. 